\title{
Time-varying Huygens' Metadevices for Parametric Wave Control
}

\author{
Mingkai Liu ${ }^{1}$, David A. Powell ${ }^{2,1}$, Yair Zarate ${ }^{1}$, Ilya V. Shadrivov ${ }^{1}$ \\ ${ }^{I}$ Nonlinear Physics Center, Research School of Physics and Engineering, the Australian National University, Canberra, ACT 2601, Australia \\ ${ }^{2}$ School of Engineering and Information Technology, University of New South Wales, Canberra, Australian Capital Territory 2610, Australia \\ Mingkai.liu@anu.edu.au
}

\begin{abstract}
Dynamic and arbitrary control of electromagnetic waves is challenging. We introduce time-varying Huygens' metadevices for efficient parametric conversion and demonstrate experimentally that the amplitude, phase, and scattering of parametric waves can be manipulated almost arbitrarily. (C) 2019 The Author(s)

OCIS codes: $260.0260,160.3918$
\end{abstract}

Huygens' metadevices provide new opportunities for highly efficient wave-front shaping and polarization manipulation using subwavelength structures [1,2]. While various static Huygens' metadevices have been demonstrated, advanced applications require that the performance of the device can be tuned dynamically to adapt to varying operating conditions. Ideally, this would require that the amplitude and phase response can be tuned not only dynamically but also independently -- for example, to achieve a pure phase tuning over $2 \pi$ or pure amplitude modulation. This is not trivial since existing tuning mechanisms of metasurfaces generally rely on either shifting the resonant frequency or the resonant linewidth, which inevitably change both the amplitude and phase simultaneously over the whole resonant bandwidth. This is a feature that is directly related to the Kramers-Kronig relation in linear systems. While there are some recent attempts to achieve independent tuning of the amplitude and phase [3], the practical realization remains very challenging as it requires independent tuning of not only the electric and magnetic responses but also the loss and gain in each meta-atom $[3,4]$.

We propose to solve this challenge by exploiting the parametric process in time-varying Huygens' metasurface (Fig.1a). When a monochromatic electromagnetic wave with a frequency $\omega_{0}$ interact with a system whose electromagnetic properities are dynamically modulated with a frequency $\Omega \ll \omega_{0}$, parametric waves of new frequencies (sidebands) $\omega_{n}=\omega_{0}+n \Omega(n \in \mathbb{Z})$ are generated in the spectral domain. While most previous studies focused on optically large systems, the effects of dynamic modulation in subwavelength systems, e.g. resonant particles and metasurfaces, became a subject of interest recently due to their potential for device-miniaturization [5, $6]$.

(a)

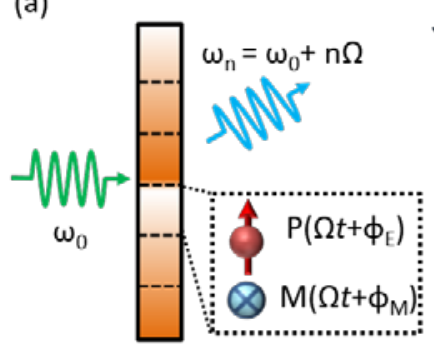

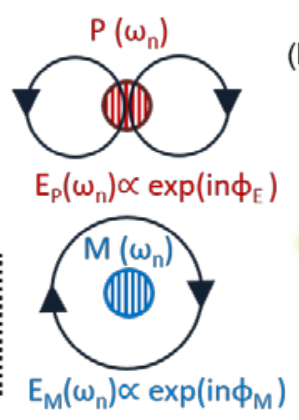

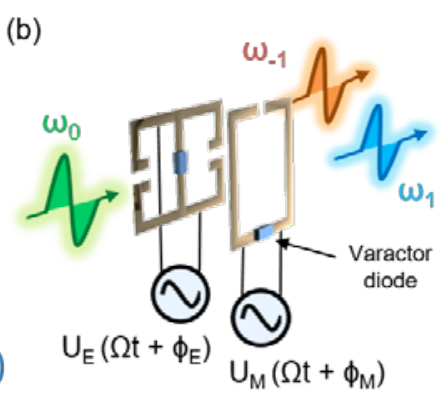

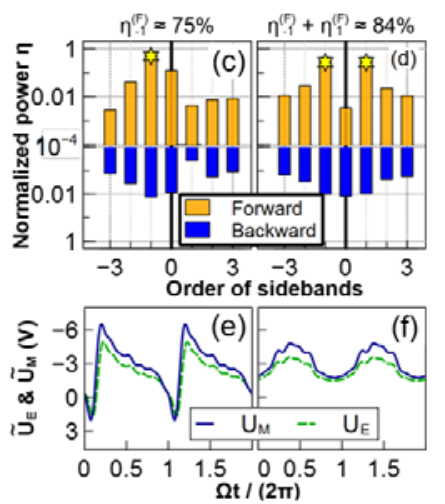

Fig. 1. (a) Schematic of time-varying Huygens' metadevices. Each unit consists of electric and magnetic metaatoms that are modulated periodically in time. The modulation phase $\phi_{\mathrm{E}(M)}$ has a gauge freedom that can be imprinted to the scattered sideband $\omega_{n}$ as $\propto \exp \left(\operatorname{in} \phi_{\mathrm{E}(M)}\right)$. The parities of the scattered fields from the electric and magnetic meta-atoms are largely preserved as long as the modulation frequency is sufficiently low. (b) Realization of a time-varying Huygens' unit composed of electric and magnetic resonators, in which varactor diodes are used as voltage-tunable elements. (c) and (d) Measured sideband spectra under different modulation signals (e) and (f). $\eta_{\mathrm{n}}^{F}$ is the normalized power (over the total scattered power of all sidebands including the carrier wave) of sideband $\omega_{n}$ scattered in the forward direction. The stars in (c) and (d) indicate the dominant sidebands. 

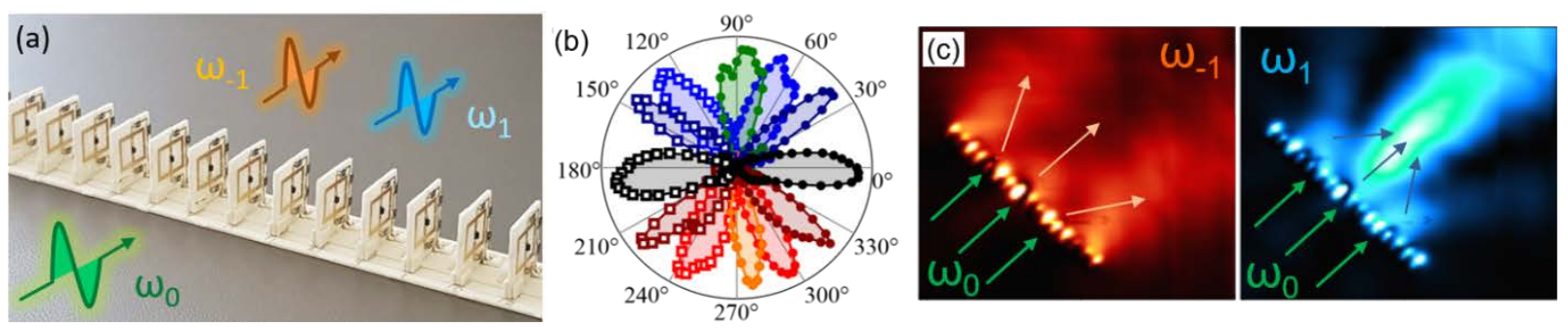

Fig. 2. (a) Photograph of a time-varying Huygens' array. (b) Polar plot of the scattering patterns of sideband $\omega_{1}$ under different linear modulation phase gradient. Each plot is normalized to its peak value. (c) The measured nearfield pattern at sidebands $\omega_{-1}$ and $\omega_{1}$, when a parabolic modulation phase pattern is applied. The time-varying Huygens' array functions as a defocusing lens at $\omega_{-1}$ and a focusing lens at $\omega_{1}$ at the same time.

One important feature of this parametric process in subwavelength scatters is that there is gauge freedom where the phases of the scattered sidebands can be controlled by the external dynamic modulation (Fig. 1b). This provides the physical foundation for independent tuning of the amplitudes and phases. By changing the modulation phase pattern along the unit cells of the metasurface, the sideband fields can be engineered in an arbitrary fashion. Importantly, since the parities of the scattered sideband field from the electric and magnetic meta-atoms are preserved (Fig. 1a), the directivity of scattering can be manipulated in an arbitrary fashion by changing the relative modulation phase of electric and magnetic meta-atoms.

In our recent work [7], we show both theoretically and experimentally that it is possible to achieve a high conversion efficiency from the incident carrier wave to the desired sidebands when combining electric and magnetic meta-atoms. We design and fabricate Huygens' units working around $4 \mathrm{GHz}$; each consisted of an electric and magnetic meta-atom that are independently modulated by an external low-frequency voltage signal (Fig. 1b). Using a genetic algorithm to optimize the waveform of the modulating voltage (Fig. 1e and 1f), we achieve a high conversion efficiency $(>75 \%)$ from the incident carrier wave to the desired sidebands, and successfully demonstrate various controlled sideband scattering, including single-sideband unidirectional scattering (Fig. 1c), double-sideband unidirectional scattering (Fig. 1d) and double-sideband bi-directional scattering. By tuning the relative modulation phase $\phi_{E}-\phi_{M}$, the directionality of the desired sidebands can be tuned to any state in between perfectly forward and perfectly backward states without sacrificing the overall efficiency.

Novel functionalities can be realized when different phase patterns are applied to an array of time-varying Huygens' units. By controlling the linear modulation phase gradient along the metasurface, as well as the relative modulation phase between the electric and magnetic meta-atoms, we achieve dynamic beam steering over the entire 360-degree range (Fig. 2b). When a parabolic phase pattern is introduced, the time-varying Huygens' metadevice allows frequency-multiplexed functionalities - it works as a concave lens at $\omega_{-1}$ and a convex lens at $\omega_{1}$ at the same time (Fig. 2c). These effects are highly nontrivial to realize using conventional tunable metadevices working in the linear regime, showing that time-varying Huygens' metadevices provide new opportunities for dynamic and arbitrary manipulation of electromagnetic waves and could be a platform for novel applications with unprecedented functionalities.

To conclude, we introduced and experimentally validated the novel concept of time-varying Huygens' metasurfaces for parametric waves. This method paved the way for constructing Huygens' metadevices whose amplitude and phase response can be tuned not only dynamically but also arbitrarily, allowing advanced functionalities that are highly nontrivial to realize using conventional linear metadevices.

[1] C. Pfeiffer, A. Grbic, "Metamaterial Huygens' Surfaces: Tailoring Wave Fronts with Reflectionless Sheets”, Phys. Rev. Lett. 110, 197401 (2013).

[2] M. Liu, Y. Zhang, X. Wang, C. Jin, "Incident-angle-insensitive and polarization independent polarization rotator", Optics Express 18, 1199012001, (2010)

[3] B. O. Zhu, K. Chen, N. Jia, L. Sun, J. Zhao, T. Jiang, and Y. Feng, "Dynamic Control of Electromagnetic Wave Propagation with the Equivalent Principle Inspired Tunable Metasurface", Sci. Rep. 4, 4971 (2014).

[4] K. Chen et al., "A Reconfigurable Active Huygens' Metalens”, Adv. Mater. 29, 1606422 (2017).

[5] Y. Hadad, D. L. Sounas, and A. Alu, "Space-Time Gradient Metasurfaces", Phys. Rev. B 92, 100304 (2015).

[6] A. Shaltout, A. Kildishev, and V. Shalaev, "Time-Varying Metasurfaces and Lorentz Non-reciprocity", Opt. Mater. Express 5, 2459 (2015).

[7] M. Liu, D. A. Powell,Y. Zarate, and I. V. Shadrivov, "Huygens' Metadevices for parametric waves”, Phys. Rev. X 8, 031077 (2018). 